\title{
Estudo radiométrico da Praia de Meaípe com rota alternativa em Guarapari-ES
}

\author{
Braga, H. $\mathrm{M}^{1}$;Ferreira, B.C.S ${ }^{1}$; Passamai, Jr.J.L. ${ }^{2 *}$; \\ 1 Departamento de Química e Física, Universidade Federal do Espírito Santo, Alegre, ES, Brasil. \\ 2 Departamento de Física, Universidade Federal do Espírito Santo, Vitória, ES, Brasil.
}

*e-mail:passamaijr@gmail.com

Resumo

O objetivo do nosso trabalho foi investigar através de uma rota alternativa a atividade radioativa na Praia de Meaípe na cidade de Guarapari-ES utilizando uma metodologia dinâmica (variação com tempo). As medidas foram tomadas em tempos distintos durante o período de (setembro de 2016 a abril de 2017). Para tanto um ciclocomputador Etrex-10 foi usado para determinar uma rota na areia da praia usada por banhistas e também um contador Geiger que mediu e armazenou os dados. Fizemos uma rota alternativa da usada nas medidas anteriores com o propósito de aferir melhor a radioatividade encontrada na areia da Praia de Meaípe.

\begin{abstract}
The objective of our work was to investigate through an alternative route the radioactive activity in Praia de Meaípe in the city of Guarapari-ES using a dynamic methodology (variation with time). Measures were taken at different times during the period from (September 2016 to April 2017). An Etrex-10 cyclocomputer was used to determine a route in the beach sand used by bathers and also a Geiger counter that measured and stored the data. We made an alternative route of the one used in the previous measurements in order to better measure the radioactivity found in the sand of Praia de Meaípe.
\end{abstract}

\section{Introdução}

O relato de Orlando et al. [1] indica uma possível relação da atividade radioativa nas praias de Guarapari com efeitos biológicos. Indiferente ao efeito da radiatividade, verificase que em geral os autores, anteriores a este estudo, descrevem as propriedades das areias utilizando amostras retiradas em um único local, de forma singular no tempo [2,3,4]. Neste trabalho foi realizada a medição do nível da atividade radioativa $(\mu \mathrm{Sv} / \mathrm{h})$ presente na praia de Meaípe variando no tempo, os estudos começaram em agosto de 2016 e foram até abril de 2017. Nesses oito meses de medidas, o intervalo entre as medidas foi de 21 dias.

Para a rota ser seguida várias vezes no mesmo caminho utilizamos as coordenadas de georreferenciamento de a tabela a seguir.
Tabela 1: Coordenadas da rota utilizada.

\begin{tabular}{|c|c|c|c|}
\hline $20^{\circ} 44^{\prime} 21.70^{\prime \prime} \mathrm{S}$ & $40^{\circ} 32^{\prime} 11.27^{\prime \prime} \mathrm{W}$ & $20^{\circ} 44^{\prime} 20.79 " \mathrm{~S}$ & $40^{\circ} 32^{\prime} 42.77^{\prime \prime} \mathrm{W}$ \\
\hline $20^{\circ} 44^{\prime} 20.93 " \mathrm{~S}$ & $40^{\circ} 32^{\prime} 12.83^{\prime \prime} \mathrm{W}$ & $20^{\circ} 44^{\prime} 21.09^{\prime \prime} \mathrm{S}$ & $40^{\circ} 32^{\prime} 44.44^{\prime \prime} \mathrm{W}$ \\
\hline $20^{\circ} 44^{\prime} 20.11^{\prime \prime S}$ & $40^{\circ} 32^{\prime} 14.35^{\prime \prime} \mathrm{W}$ & $20^{\circ} 44^{\prime} 21.26^{\prime \prime S}$ & $40^{\circ} 32^{\prime} 46.15^{\prime \prime} \mathrm{W}$ \\
\hline $20^{\circ} 44^{\prime} 19.38^{\prime \prime S}$ & $40^{\circ} 32^{\prime} 15.90^{\prime \prime} \mathrm{W}$ & $20^{\circ} 44^{\prime} 21.51^{\prime \prime S}$ & $40^{\circ} 32^{\prime} 47.82^{\prime \prime} \mathrm{W}$ \\
\hline $20^{\circ} 44^{\prime} 18.79^{\prime \prime S}$ & $40^{\circ} 32^{\prime} 17.55^{\prime \prime} \mathrm{W}$ & $20^{\circ} 44^{\prime} 21.84^{\prime \prime S}$ & $40^{\circ} 32^{\prime} 49.51^{\prime \prime} \mathrm{W}$ \\
\hline $20^{\circ} 44^{\prime} 18.27 " \mathrm{~S}$ & $40^{\circ} 32^{\prime} 19.24^{\prime \prime} \mathrm{W}$ & $20^{\circ} 44^{\prime} 22.36^{\prime \prime S}$ & $40^{\circ} 32^{\prime} 51.14^{\prime \prime} \mathrm{W}$ \\
\hline $20^{\circ} 44^{\prime} 17.88^{\prime \prime S}$ & $40^{\circ} 32^{\prime} 20.93^{\prime \prime} \mathrm{W}$ & $20^{\circ} 44^{\prime} 23.51^{\prime \prime S}$ & $40^{\circ} 32^{\prime} 53.34^{\prime \prime} \mathrm{W}$ \\
\hline $20^{\circ} 44^{\prime} 17.55^{\prime \prime S}$ & $40^{\circ} 32^{\prime} 22.68^{\prime \prime} \mathrm{W}$ & $20^{\circ} 44^{\prime} 24.41 " \mathrm{~S}$ & $40^{\circ} 32^{\prime} 54.78^{\prime \prime} \mathrm{W}$ \\
\hline $20^{\circ} 44^{\prime} 17.30^{\prime \prime S}$ & $40^{\circ} 32^{\prime} 24.40^{\prime \prime} \mathrm{W}$ & $20^{\circ} 44^{\prime} 25.39^{\prime \prime} \mathrm{S}$ & $40^{\circ} 32^{\prime} 56.19^{\prime \prime} \mathrm{W}$ \\
\hline $20^{\circ} 44^{\prime} 17.21 " \mathrm{~S}$ & $40^{\circ} 32^{\prime} 26.13^{\prime \prime} \mathrm{W}$ & $20^{\circ} 44^{\prime} 26.38^{\prime \prime S}$ & $40^{\circ} 32^{\prime} 57.53^{\prime \prime} \mathrm{W}$ \\
\hline $20^{\circ} 44^{\prime} 17.37 " \mathrm{~S}$ & $40^{\circ} 32^{\prime 2} 27.89^{\prime \prime} \mathrm{W}$ & $20^{\circ} 44^{\prime} 27.51^{\prime \prime S}$ & $40^{\circ} 32^{\prime} 58.75^{\prime \prime} \mathrm{W}$ \\
\hline $20^{\circ} 44^{\prime} 17.50^{\prime \prime S}$ & $40^{\circ} 32^{\prime} 29.64^{\prime \prime} \mathrm{W}$ & $20^{\circ} 44^{\prime} 28.28^{\prime \prime S}$ & $40^{\circ} 33^{\prime} 0.23^{\prime \prime} \mathrm{W}$ \\
\hline $20^{\circ} 44^{\prime} 17.62^{\prime \prime S}$ & $40^{\circ} 32^{\prime} 31.38^{\prime \prime} \mathrm{W}$ & $20^{\circ} 44^{\prime} 28.97^{\prime \prime S}$ & $40^{\circ} 33^{\prime} 1.80^{\prime \prime} \mathrm{W}$ \\
\hline $20^{\circ} 44^{\prime} 17.77 " \mathrm{~S}$ & $40^{\circ} 32^{\prime} 33.07^{\prime \prime} \mathrm{W}$ & $20^{\circ} 44^{\prime} 29.75^{\prime \prime S}$ & $40^{\circ} 33^{\prime} 3.35^{\prime \prime} \mathrm{W}$ \\
\hline $20^{\circ} 44^{\prime} 17.99^{\prime \prime S}$ & $40^{\circ} 32^{\prime} 34.77^{\prime \prime} \mathrm{W}$ & $20^{\circ} 44^{\prime} 30.78^{\prime \prime S}$ & $40^{\circ} 33^{\prime} 4.71^{\prime \prime} \mathrm{W}$ \\
\hline $20^{\circ} 44^{\prime} 18.44 " \mathrm{~S}$ & $40^{\circ} 32^{\prime} 36.38^{\prime \prime} \mathrm{W}$ & $20^{\circ} 44^{\prime} 31.76 " \mathrm{~S}$ & $40^{\circ} 33^{\prime} 6.08^{\prime \prime} \mathrm{W}$ \\
\hline $20^{\circ} 44^{\prime} 19.17^{\prime \prime S}$ & $40^{\circ} 32^{\prime} 38.50^{\prime \prime} \mathrm{W}$ & $20^{\circ} 44^{\prime} 32.64^{\prime \prime S}$ & $40^{\circ} 33^{\prime} 7.57^{\prime \prime} \mathrm{W}$ \\
\hline $20^{\circ} 44^{\prime} 19.66^{\prime \prime S}$ & $40^{\circ} 32^{\prime} 40.15^{\prime \prime} \mathrm{W}$ & $20^{\circ} 44^{\prime} 33.23 " \mathrm{~S}$ & $40^{\circ} 33^{\prime} 9.16^{\prime \prime} \mathrm{W}$ \\
\hline $20^{\circ} 44^{\prime} 20.22 " \mathrm{~S}$ & $40^{\circ} 32^{\prime} 41.11^{\prime \prime} \mathrm{W}$ & & \\
\hline
\end{tabular}




\section{Resultados e Discussões}

$\mathrm{Na}$ figura 01, registramos as três primeiras medidas realizadas entre o mês de agosto de 2016 até abril de 2017 e obtivemos o seguinte gráfico.

Fig. 01. Gráfico das três primeiras medidas

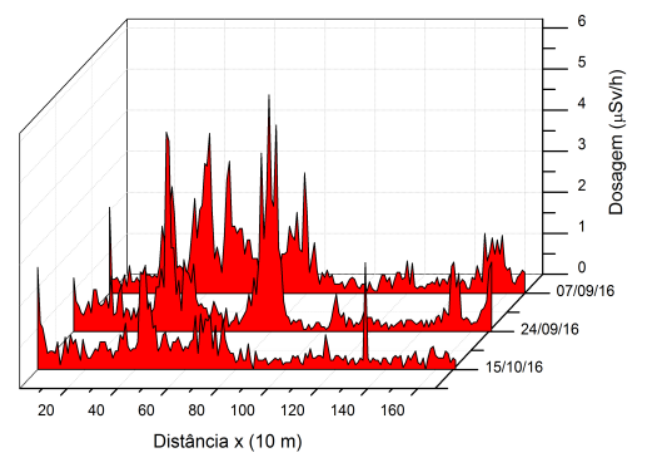

Á direita do gráfico acima temos 3 datas que foram feitas as medidas, a primeira foi feita em 07/09/16 a segunda em 24/09/16 e a terceira em 15/10/16.

Durante a primeira medida verificamos uma dose de aproximadamente $3,89(\mu \mathrm{Sv} / \mathrm{h}) \mathrm{em}$ $410 \mathrm{~m}$, na segunda medida verificamos uma dose de $5,02(\mu \mathrm{Sv} / \mathrm{h})$ em $820 \mathrm{~m}$, na terceira medida verificamos uma dosagem 3,98 $(\mu \mathrm{Sv} / \mathrm{h})$ em $1320 \mathrm{~m}$.

Realizamos mais medidas e obtivemos o gráfico a seguir.

Fig. 02. Gráfico das três últimas medidas.

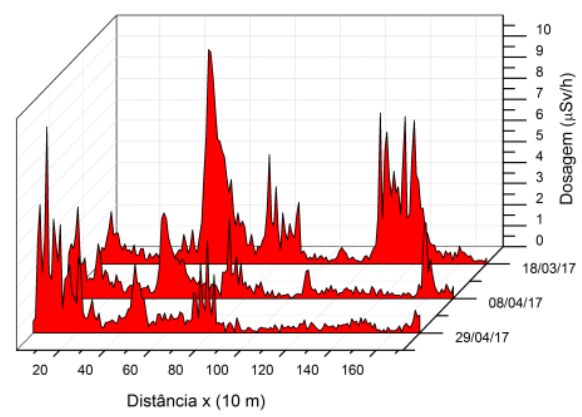

A medida do dia 18/03/17 mostra a dosagem de 10,17 $(\mu \mathrm{Sv} / \mathrm{h})$ em $490 \mathrm{~m}$, na segunda medida verificamos uma dose de $4,34(\mu \mathrm{Sv} / \mathrm{h})$ em $60 \mathrm{~m}$, na terceira medida verificamos uma dosagem 9,77 $(\mu \mathrm{Sv} / \mathrm{h})$ próximo de $70 \mathrm{~m}$.

\section{Conclusões}

Nossas medições neste trabalho mostram que a radiação nesta praia varia de intensidade com o tempo e se desloca no espaço continuamente nesta praia.

O ponto a ser ressaltado aqui é de que análises instantâneas de amostras de areia não caracterizam bem a Praia de Meaípe, uma vez que o sistema é dinâmico.

Somente uma análise temporal com base em 1 ano pode fornecer valores médios da atividade radioativa na Praia de Meaípe.

\section{Referências}

[1] Orlando, M T D, et al. Correlation between Breast Cancer and Radiation Level of Guarapari City - ES, Blucher Proceedings ISSN: 2358-2359 V 01, (2014) n 02 DOI:10.5151/phypro-ecfa-020

[2] Fujinami, N.; T. Koga, T.; Morishima. H. External Expousure Rates From Terrestrial Radiantion At Guarapari And Meaipe In Brazil. (1999).

[3] Vasconcelos, D.C, Et Al. Modelling Natural Radioactivity In Sand Beaches Of Guarapari, Espírito Santo State, Brazil. (2013).

[4] Calheiro, D.S.; Passamai. Jr.,J.L. Estudo da Radiação na areia da Praia da Areia Preta. (2016). DOI: $10.5151 /$ phypro-vii-efa-035

Agradecimentos.

Comissão Nacional de Energia Nuclear (CNEN), ao CNPQ e a Fundação de Amparo à Pesquisa e Inovação do Espírito Santo (FAPES) (projetos 098/2019 e 270/2019). 